\title{
Carrot/Jilin Ginseng/Licorice Root/Tangerine Peel/Soy Beverage
}

National Cancer Institute

\section{Source}

National Cancer Institute. Carrot/lilin Ginseng/Licorice Root/Tangerine Peel/Soy

Beverage. NCI Thesaurus. Code C90538.

A soy-based powdered nutritional supplement drink containing carrot, jlin ginseng, licorice root and tangerine peel with potential antioxidant, immunomodulating and protective activities. Besides vitamin C, E and other phytochemicals, carrot/jilin ginseng/licorice root/tang erine peel/soy beverage contains a high amount of soy protein. This beverage may have a beneficial effect on overall nutrition and the immune system. 